\title{
Experten bewerten Karies als nicht übertragbar
}

Im Vorfeld des diesjährigen Kongresses der Europäischen Gesellschaft für Kariesforschung (ORCA) in Brüssel fand mit Unterstützung von Colgate/CP GABA eine Symposiumsdebatte statt unter dem Motto: „Sollte Karies als eine nichtübertragbare Erkrankung betrachtet werden?“ In einer anschließenden Teilnehmerbefragung sprach sich die überwiegende Mehrheit der Dentalexperten dafür aus, Karies als nicht übertragbare Erkrankung einzustufen. Dieser Wandel im Verständnis von Karies ist ein wichtiger Schritt zur Verbesserung der Kariesprävention und -behandlung. Der Leiter der Symposiumsdebatte und Co-Vorsitzender der Alliance for a Cavity-Free Future (ACFF), Professor Nigel Pitts vom King،s College London, erinnerte daran, dass Karies zu den am besten vermeidbaren chronischen Erkrankungen zählt. Diese Erkenntnis wird bei der Entwicklung effizienterer Maßnahmen zur Kariesprävention, -behandlung und -überwachung helfen. Die ZuckersäurenNeutralisator-Technologie von elmex nutzt diesen Ansatz durch die klinisch ge- testete Wirkung von Arginin. Das Arginin neutralisiert den $\mathrm{pH}$-Wert in der Plaque und fördert somit eine gesündere Bakterienflora. Der hochwirksame Kariesschutz der fluoridhaltigen Zahnpasta mit der PRO-ARGIN-Technologie entsteht durch den direkten Einfluss auf die dentale Plaque. Gefährliche Zuckersäuren werden neutralisiert, bevor sie den Zahnschmelz angreifen können. Durch den Anstieg des pH-Werts in der Plaque wird das gesunde Gleichgewicht im dentalen Biofilm wiederhergestellt und damit nachweislich die Entstehung neuer Kariesläsionen signifikant reduziert: Bis zu 17\% laut einer 2013 publizierten klinischen 2-Jahres-Studie (Kraivaphan et al.) und bis $\mathrm{zu}$ $20 \%$ in der jüngsten Untersuchung von $\mathrm{Li}$ $X$ et al. (2015) bei der täglichen Verwendung der Zahnpasta KARIESSCHUTZ PROFESSIONAL im Vergleich zu einer handelsüblichen fluoridhaltigen Zahnpasta.

Nach einer Pressemitteilung der

CP GABA GmbH, Hamburg 